\title{
Education empowers women to reach their personal fertility target, regardless of what the target is
}

\section{Wolfgang Lutz*}

When we study the effects of education on fertility, it is necessary to distinguish between setting a target for family size, and the ability to actually reach this target. In highly developed countries with low fertility, the interplay of these two independent forces can result in rather complex patterns of education-fertility associations. My main hypothesis here is that education typically empowers women (and couples) to reach their personal target for family size, regardless of what the target is. If the educated women in a given country want to have a family size that exceeds the average fertility rate in that country, the fertility rate of highly educated women may be higher than the average fertility rate. The empirical data do not yet show many examples of such cases. In highly developed countries with high levels of gender equity, such as the Nordic countries, some U-shaped patterns - in which the least educated and the most educated women have somewhat higher fertility than the women with medium levels of education - have been observed. In the future, such patterns may appear in more countries. But as I argue in this note, this pattern should not be seen as an indication that education is no longer a key determinant of fertility.

The effects of education on health and survival are more straightforward than they are on fertility, because the goals in health and survival are always in one direction. Irrespective of their stage of socioeconomic development, virtually all people at any given point in time want to be in good rather than in bad health, and want to avoid the premature deaths of themselves and their family members. This is also the reason why empirical research for virtually all settings shows that compared to their less educated counterparts, better educated women have lower child mortality, and better educated men and women have higher life expectancy. There have been a number of studies on the relationship between health/mortality and education, and the findings of this research leave little doubt that the many dimensions of empowerment that result from education have real and causal effects on health and survival. (Baker et al. 2011; Lutz and Skirbekk 2014). There is clear evidence that all learning experiences,

\footnotetext{
* Wolfgang Lutz, Wittgenstein Centre for Demography and Global Human Capital (IIASA, VID/ÖAW, WU), International Institute for Applied Systems Analysis, Schlossplatz 1, 2361 Laxenburg, Austria Email: lutz@iiasa.ac.at
} 
including learning experiences through formal education, change our brains for the rest of our lives (Kandel 2007). Education tends to enhance our time horizons, and to influence our health-related behaviors in a multitude of ways that are mostly conducive to health and survival.

In terms of fertility, the effect of female education during the process of demographic transition, when family size gradually moves from a high and mostly uncontrolled condition to a low and planned condition, is also clear and straightforward. During this transition, it is evident that better educated women generally want to have fewer children, and that they find increasingly effective ways to ensure that they have fewer children. The desired family size tends to be smaller for better educated women than for their less educated counterparts, because the former group are more concerned about the quality of children. Thus, highly educated women tend to seek to maximize the life chances of their children by having fewer children who receive high levels of nutrition and education. Under poor sanitary conditions and in the absence of efficient health care services, having a large number of pregnancies also carries significant health risks for the mother. Under such conditions, better educated women are typically aware of these health risks, and may try to limit their number of pregnancies in the interests of their own health. Hence, in countries undergoing the demographic transition, there is a consistent and strong negative association between female education and desired family size. In addition, many studies have shown that education empowers women to achieve their lower goals, as they are often able to overcome resistance from their husband or extended family, and to access relevant information and reproductive health services (Fuchs and Goujon 2014). Based on data from DHS surveys in 30 African countries, John Bongaarts (2010) found that educational levels are systematically positively associated with the demand for and the use of contraception, and are negatively associated with desired family size. These two factors together clearly result in lower actual fertility among better educated women.

For highly developed countries with low fertility, there are good reasons to assume that the generally empowering effect of education also holds, as education places women in a better position to organize their lives according to their intentions. In terms of family planning, survey evidence shows that compared to less educated women, highly educated women are better able to realize their stated fertility plans; i.e., to determine the quantum and the timing of their births, and to have fewer unplanned births (Musick et al. 2009). Hence, if the fertility targets of better educated women in highly developed countries were consistently lower than those of less educated women, as they tend to be in countries undergoing the demographic transition, we would undoubtedly find a strong and consistent negative association between education and fertility. However, the data show that the fertility targets of better educated women are not consistently lower than those of less educated women in countries where fertility levels are around or below the replacement level (Sobotka et al. 2015). Hence, the key to understanding future trends in fertility among highly educated women in low fertility countries lies in understanding their fertility targets. 
The figure below offers a possible conceptualization of the translation from the personal ideal family size to fertility targets and their realization in the form of actual births. The figure is a modification of the conceptual framework introduced in the context of the "low fertility trap hypothesis" of Lutz et al. (2006). This hypothesis distinguishes between the different aspects of fertility, ranging from ideal family size to actual births (as shown on the left side of the graph); and assumes that there are different forms of feedback from actual fertility experienced at the societal level, which is in turn based on the ideals and the realization of these ideals at the individual level. In particular, the hypothesis states that a high prevalence of onechild families in a society will influence the norms of the next generation; i.e., that the observed reality may be taken as the new norm, which could cause the ideal family size to fall well below two children per couple. While there is little empirical evidence of such a pattern in Europe, where the two-child norm still seems very strong; in East Asia, and particularly in the Chinese urban areas where a one-child policy had been enforced for several decades, it appears that a new one-child norm has become dominant, even in places where couples are now allowed to have two children (Basten and Jiang 2015).

The figure presented here does not include the feedback loops; instead, it only shows the different aspects of fertility (on the left-hand side of the figure), and some selected mechanisms that influence these aspects, which can in turn be seen as having been influenced by education. The personal ideal family size is seen here as fully exogenous and determined by culture and other forces, which may include the forces described in the low fertility trap hypothesis. Next, the personal ideal family size is translated into a more concrete fertility target. This target has already been shaped by the individual's awareness of certain trade-offs and of other life goals that compete with fertility desires. For better educated women in particular, there is often a clear conflict between the desire to have a successful professional career and the desire to have children. How this conflict is resolved depends greatly on the welfare system of the society, including on the availability of child care and on the degree of gender equity. Hence, it is hard to come up with a general assessment of how higher education contributes to the resolution of this conflict. For example, the empirical data for the 1966-70 birth cohorts show that highly educated women were much more likely to remain childless in Germany than in France (Sobotka et al. 2015). Another possible negative effect of education on fertility operates through the choice of partner. For example, in some societies, and particularly in East Asia, highly educated women do not want to marry a less educated man, and may therefore have problems finding an appropriate spouse for establishing a family.

The formation of the personal fertility target is thus very complex and hard to predict; and it is equally difficult to find a systematic effect of education on this aspect of fertility. However, the effect of education on the realization of the target, whatever it happens to be, is much clearer. I am confident that now and in the future better educated women will be more empowered to manage their lives in ways that allow them to actually meet their targets. 
Figure 1:

A simple conceptualization of the possible effects of higher education on the decision-making processes that lead to actual fertility

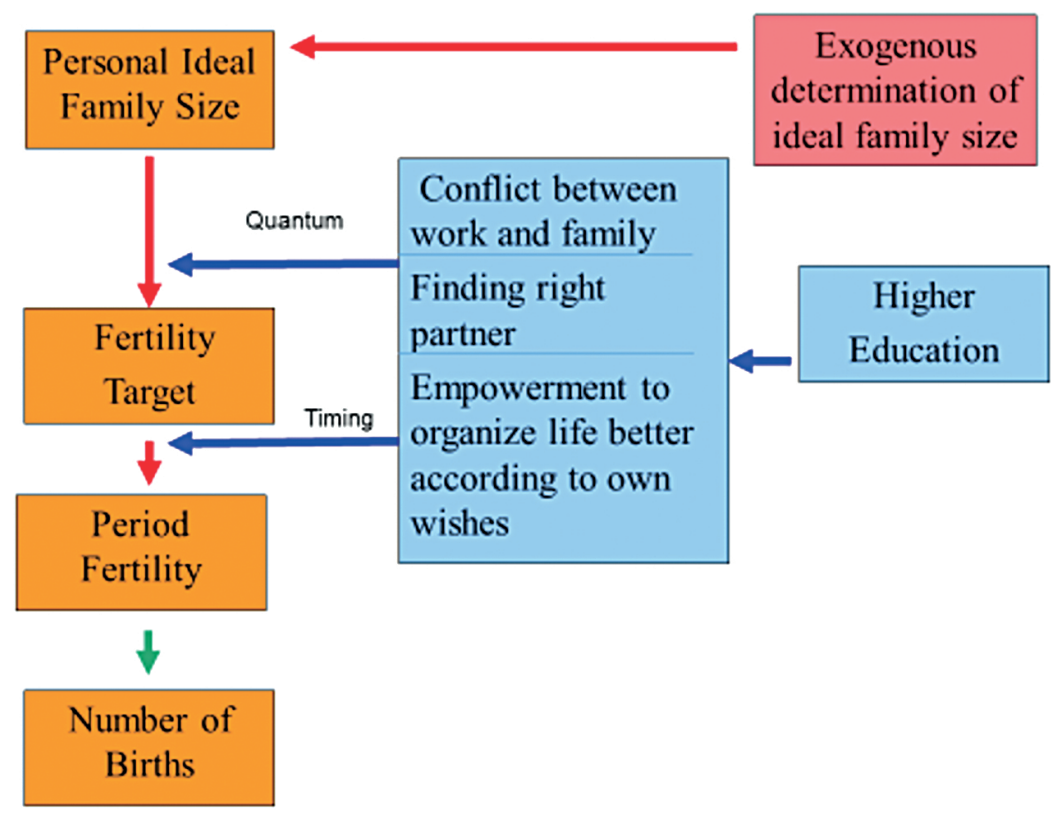

Hence, in sum, my answer to the question of whether in the future better educated women in countries with low fertility will have lower or higher fertility has two parts: (a) We cannot know the effect of education on fertility targets because these targets depend on local and cultural conditions, and may change over time. (b) I am, however, quite confident that education affects the ability of women to realize their fertility intentions; and that in the future, better educated women will be able to better reach their targets.

Therefore, if we assume that in Europe the fertility targets of educated women will be somewhere around two children, we can expect to see slight increases in the actual fertility of better educated women, who, on average, still have much lower fertility than their less educated counterparts.

\section{References}

Baker, D. P., Leon, J., Smith Greenaway, E. G., Collins, J., and Movit, M. 2011. The education effect on population health: A reassessment. Population and Development Review 37(2): 307-332, DOI: 10.1111/j.1728-4457.2011.00412.x.

Basten, S. and L. Jiang. 2015. Fertility in China: An uncertain future. Population Studies 69(sup1): S97-S105. 
Bongaarts, J. 2010. The causes of educational differences in fertility in Sub-Saharan Africa. Vienna Yearbook of Population Research 8: 31-50,

DOI: 10.1553/populationyearbook2010s31.

Fuchs, R. and Goujon, A. 2014. Future fertility in high fertility countries. In World population and human capital in the 21st century, eds Lutz, W., Butz, W. P. and KC, S., 147-225. Oxford: Oxford University Press, http://ukcatalogue.oup.com/product/9780198703167.do.

Kandel, E. R. 2007. In search of memory: The emergence of a new science of mind. New York: W.W. Norton \& Co.

Lutz, W. 2008. Has Korea's fertility reached the bottom? The hypothesis of a 'low Fertility Trap' in parts of Europe and East Asia. Asian Population Studies 4(1): 1-4, DOI: 10.1080/17441730801963110.

Lutz, W. and Skirbekk, V. 2014. How education drives demography and knowledge informs projections. In World population and human capital in the 21 st century, eds Lutz, W., Butz, W. P. and KC, S., 14-38. Oxford: Oxford University Press, http://ukcatalogue.oup.com/product/9780198703167.do.

Lutz, W., Skirbekk, V., and Testa, M. R. 2006. The low-fertility trap hypothesis: Forces that may lead to further postponement and fewer births in Europe. Vienna Yearbook of Population Research 4: 167-192, http://www.jstor.org/stable/10.2307/23025482.

Musick, K., P. England; S. Edgington, and N. Kangas. 2009. Education differences in intended and unintended fertility. Social Forces 88(2): 543-572.

Sobotka, T., K. Zeman, M. Potančoková, J. Eder, Z. Brzozowska, É. Beaujouan, and A. Matysiak. 2015. European fertility datasheet 2015. Vienna Institute of Demography/Wittgenstein Centre for Demography and Global Human Capital (IIASA, VID/ÖAW, WU), http://www.fertilitydatasheet.org/. 
